\title{
Harmonizing evidence-based practice, implementation context, and implementation strategies with user-centered design: a case example in young adult cancer care
}

\section{Emily R Haines ( $\nabla$ ehaines@email.unc.edu )}

University of North Carolina at Chapel Hill https://orcid.org/0000-0001-7208-5937

\section{Alex Dopp \\ RAND Corp}

Aaron R. Lyon

University of Washington

Holly O. Witteman

Universite Laval

Miriam Bender

University of California Irvine

\section{Gratianne Vaisson}

Universite Laval

\section{Danielle Hitch}

Deakin University

\section{Sarah Birken}

University of North Carolina at Chapel Hill

\section{Methodology}

Keywords: user-centered design, human-centered design, context, evidence-based practice implementation, designing implementation strategies, contextual appropriateness, EBP redesign, stakeholder engagement, adaptation

Posted Date: January 16th, 2020

DOI: https://doi.org/10.21203/rs.2.20978/v1

License: (c) (1) This work is licensed under a Creative Commons Attribution 4.0 International License. Read Full License 
Version of Record: A version of this preprint was published at Implementation Science Communications on April 26th, 2021. See the published version at https://doi.org/10.1186/s43058-021-00147-4. 


\section{Abstract}

Background. Attempting to implement evidence-based practices in contexts for which they are not wellsuited may compromise their fidelity and effectiveness or burden users (e.g., patients, providers, healthcare organizations) with elaborate strategies intended to force implementation. To improve the fit between evidence-based practices and contexts, implementation science experts have called for methods for adapting evidence-based practices and contexts, and tailoring implementation strategies; yet, methods for considering the dynamic interplay among evidence-based practices, contexts, and implementation strategies remain lacking. We argue that harmonizing the three can be accomplished with User-Centered Design, an iterative and highly stakeholder-engaged set of principles and methods.

Methods. This paper presents a case example in which we used User-Centered Design methods and a three-phase User-Centered Design process to design a care coordination intervention for young adults with cancer. Specifically, we used usability testing to redesign an existing evidence-based practice (i.e., patient-reported outcome measure that served as the basis for intervention) to optimize usability and usefulness, an ethnographic user and contextual inquiry to prepare the context (i.e., comprehensive cancer center) to promote receptivity to implementation, and iterative prototyping workshops with a multidisciplinary design team to design the care coordination intervention and anticipate implementation strategies needed to enhance contextual fit.

Results. Our User-Centered Design process resulted in the Young Adult Needs Assessment and Service Bridge (NA-SB), including a patient-reported outcome measure redesigned to promote usability and usefulness and a protocol for its implementation. By ensuring NA-SB directly responded to features of users and context, we designed NA-SB for implementation, potentially minimizing the strategies needed to address misalignment that may have otherwise existed. Furthermore, we designed NA-SB for scale-up ; by engaging users from other cancer programs across the country to identify points of contextual variation which would require flexibility in delivery, we created a tool not overly tailored to one unique context.

Conclusions. User-Centered Design can help maximize usability and usefulness when designing evidence-based practices, preparing contexts, and informing implementation strategies- in effect, harmonizing evidence-based practices, contexts, and implementation strategies to promote implementation and effectiveness.

\section{Contributions To The Literature}

- Novel approaches are needed to harmonize evidence-based practices, the contexts in which they are implemented, and the implementation strategies intended to facilitate their implementation, thus minimizing burden on patients, providers, and healthcare organizations while optimizing implementation. 
- User-Centered Design can be leveraged by implementation scientists to: (a) optimize EBP design to improve key determinants of implementation like usability and usefulness, (b) prepare context to promote receptivity toward EBPs (e.g., modifying workflows to accommodate EBP), and (c) select or design implementation strategies which increase the contextual appropriateness of an EBP.

\section{Background}

Evidence-based practice (EBP) implementation is often challenged by poor fit between EBPs and their implementation contexts (i.e., the "set[s] of characteristics and circumstances that consist of active and unique factors, within which the implementation is embedded")[1, 2]. Use of an EBP (i.e., intervention with proven efficacy and effectiveness[3]) in a context for which it is not well-suited can compromise its effectiveness and burden users (e.g., patients, providers, healthcare organizations) with elaborate strategies intended to force implementation. However, EBPs are seldom designed to address the nuances of multiple, varying, complex, and changing practice contexts[1]. To accommodate nuanced contexts, EBP developers may produce increasingly complex EBPs[4], resulting in EBPs "that are ultimately too expensive, impractical, or even impossible to construct within real-world constraints"[5]. Despite consistent recognition that there is no implementation without some adaptation, few methods exist to inform systematic EBP adaptation[6].

Implementation scientists have identified various EBP characteristics that influence implementation[7]; such evidence may inform efforts to adapt EBPs to improve implementation. However, the relation between EBP characteristics and implementation outcomes varies across EBPs and contexts[7], and the same EBP may demonstrate varying degrees of effectiveness in achieving the desired patient outcomes across different contexts[8]. All of this suggests that an EBP's implementation and effectiveness are inextricably linked to context. Methods for considering the dynamic interplay between EBP and context have not been well articulated[6, 9].

To address discordance between EBPs and contexts, implementation scientists often turn to implementation strategies - i.e., "methods or techniques used to enhance the adoption, implementation, and sustainability" of EBPs[10, 11]. However, a "more is better" approach to deploying implementation strategies to compensate for poor EBP-context fit may burden EBP users. Moreover, implementation strategies have shown only modest effect sizes[12]. These findings may be in part due to an insufficient consideration of key determinants, such as contextual appropriateness, when selecting or designing implementation strategies[13]. To this end, implementation scientists have called for methods for tailoring implementation strategies to EBPs and contexts[13, 14].

Rather than deploying cumbersome EBPs or implementation strategies to improve EBP-context fit, implementation scientists should seek to harmonize EBPs, contexts, and strategies (i.e., design each with respect to the other two). An analogy (Fig. 1) helps illustrate this harmonization: in embroidery, decisions about fabric, needle, or thread are interdependent. For example, a lightweight fabric and thin thread demand a smaller needle; using a large needle may damage lightweight fabric and thin thread. Likewise, 
too-thin thread may break if used with a thick needle or heavy fabric. Depending on thread count, fabric may require stabilizer or alteration before embroidering. Similarly, an EBP (i.e., the thread), context (i.e., the fabric), and implementation strategies (i.e., the needle) should be harmonized to minimize user burden and optimize implementation.

There is a critical need for the development of "relational, and dynamic approaches to theorising the complex interplay between the characteristics of interventions, the activities of implementers, and the properties of variable broader contexts"[15]. Indeed, advancing methods for harmonizing EBPs, contexts, and implementation strategies has been articulated as a priority for implementation research[7, 16]. Here, we argue that such harmonizing can be accomplished with User-Centered Design (UCD), an iterative and highly stakeholder-engaged process for designing EBPs, preparing contexts, and informing implementation strategies. To demonstrate, we present a case example in young adult cancer care. Specifically, we describe a three-phase UCD process - (1) usability testing (optimizing the thread - i.e., EBP), (2) ethnographic contextual inquiry (understanding and preparing the fabric - i.e., context), (3) prototyping with a multidisciplinary design team (threading the needle - i.e., designing EBP and implementation strategies) - to design a care coordination intervention for implementation in a comprehensive cancer center. In this article, we focus on describing the methods used in our project; more detailed results will be presented in future publications.

\section{User-Centered Design}

UCD is an iterative and highly stakeholder-engaged process for creating products which are directly responsive to their intended users and users' contexts[17]. Primary goals of UCD are improving EBP usability (ease with which it can be successfully used[18]) and usefulness (extent to which it does what it is intended to do[19]). Usability and usefulness are proximal determinants of perceptual implementation outcomes (i.e., acceptability, feasibility, and appropriateness; e.g., usability promotes acceptability) through which they also influence distal behavioral implementation outcomes (e.g., penetration, reach, sustainment; e.g., acceptability promotes reach)[20].

Most UCD definitions and frameworks share a common set of principles that contribute to harmonizing EBPs, contexts, and implementation strategies: (1) refining EBPs based on user input to optimize usability and usefulness[21]; (2) engaging prospective users to achieve a nuanced understanding of context; and (3) a multidisciplinary design team collaborating to produce design and implementation prototypes. Together, these domains comprise an iterative cycle in which an EBP's design and implementation strategies are refined until optimized for a given context[22]. Within each of these domains, UCD offers myriad methods[21] and strategies[23] for harmonizing EBPs, contexts, and implementation strategies (summarized in Table 1). Although some of UCD's discrete methods and principles resemble those traditionally used in implementation science (e.g., stakeholder engagement), UCD is unique in its offering of an extensive suite of methods that may be leveraged to refine EBPs, contexts, and implementation strategies. We present UCD as one promising set of approaches implementation scientists may consider drawing upon. 
Table 1

Applications of UCD in Implementation Science

\begin{tabular}{|c|c|c|}
\hline Construct & Definition & What UCD offers \\
\hline $\begin{array}{l}\text { Evidence-based } \\
\text { practice } \\
\text { (the thread) }\end{array}$ & $\begin{array}{l}\text { Interventions with } \\
\text { demonstrated efficacy and } \\
\text { effectiveness including } \\
\text { programs, actions, processes, } \\
\text { policies, and guidelines[3] }\end{array}$ & $\begin{array}{l}\text { - Selecting EBPs that are appropriate for users } \\
\text { and their context (e.g., by leveraging UCD } \\
\text { measures of usability such as the System } \\
\text { Usability Scale[60]) } \\
\text { - Redesigning EBPs to better fit users and their } \\
\text { context (e.g., conducting usability test or } \\
\text { heuristic evaluation to identify an EBP's design } \\
\text { limitations) }\end{array}$ \\
\hline $\begin{array}{l}\text { Context } \\
\text { (the fabric) }\end{array}$ & $\begin{array}{l}\text { Set of characteristics and } \\
\text { circumstances that consist of } \\
\text { active and unique factors, } \\
\text { within which the } \\
\text { implementation is embedded } \\
\text { including: } \\
\text { - Inner (i.e., intra- } \\
\text { organizational) context[75] } \\
\text { - Outer (i.e., extra- } \\
\text { organizational) context[75] }\end{array}$ & $\begin{array}{l}\text { - Assessing context (e.g., conducting } \\
\text { ethnography or developing user experience } \\
\text { models) } \\
\text { - Preparing context to promote receptivity to } \\
\text { EBP (e.g., using workflow mapping to modify } \\
\text { workflow to accommodate EBP } \\
\text { implementation) }\end{array}$ \\
\hline $\begin{array}{l}\text { Implementation } \\
\text { strategies } \\
\text { (the needle) }\end{array}$ & $\begin{array}{l}\text { Methods or techniques used to } \\
\text { enhance the adoption, } \\
\text { implementation and } \\
\text { sustainability of an EBP[10] }\end{array}$ & $\begin{array}{l}\text { - Anticipating needed implementation } \\
\text { strategies based on context assessment (e.g., } \\
\text { conducting design workshops to identify areas } \\
\text { where fit between EBP and context is low and } \\
\text { problem-solve accordingly) } \\
\text { - Selecting strategies that are appropriate given } \\
\text { EBP and context (e.g., using the Cognitive } \\
\text { Walkthrough for Implementation Strategies[76] } \\
\text { to assess strategy usability) } \\
\text { - Tailoring/designing strategies for EBP and } \\
\text { context (e.g., by conducting iterative co-creation } \\
\text { sessions with users) }\end{array}$ \\
\hline
\end{tabular}

\section{Methods}

Case example: implementation of a cancer needs assessment

\section{Background and project objectives}

Each year, more than 20,000 young adults between the ages of 18 and 30 are diagnosed with cancer[25]; many of them do not receive services to meet the range of needs they experience during and after cancer treatment[26-30]. Young adults' unmet needs result in negative outcomes, including higher distress[27, 28], poorer health-related quality of life[31], and higher physical symptom burden[26]. Despite the complexity and scope of their needs, young adults often do not use potentially beneficial services/resources, even when access is not an issue[32-34]. This disconnect between young adult needs and their use of existing services/resources suggests the need for a care coordination model that (1) effectively assesses young adults' multifaceted, age-specific, individual, and dynamic needs, and (2) uses that information to efficiently connect them to services/resources. 
A substantial step toward this care coordination model was the development of the first multidimensional measure of unmet needs designed specifically for adolescents and young adults: the Cancer Needs Questionnaire - Young People (CNQ-YP)[35, 36]. However, limitations to the usability and usefulness of patient-reported outcome measures like the CNQ-YP (e.g., length; wording ambiguity; redundancy or missing content; lack of connection between identified needs and follow-up actions) have frustrated their real-world implementation and effect on patient outcomes[37, 38]. In this project, we used UCD to redesign the CNQ-YP to optimize its usability and usefulness and prepare for its implementation in the University of North Carolina's Lineberger Comprehensive Cancer Center (hereafter, Lineberger). Our UCD process (Table 2, Fig. 2) produced the Needs Assessment and Service Bridge (NA-SB), a care coordination intervention for young adults with cancer. All procedures were approved by the University of North Carolina's Institutional Review Board.

Table 2

Data Collection Summary

\begin{tabular}{|c|c|c|}
\hline UCD Aim & Method & Deliverable \\
\hline $\begin{array}{l}\text { Review and refine EBP prototype } \\
\text { (the thread) }\end{array}$ & $\begin{array}{l}\text { Usability Testing } \\
\text { - Young adult Survey } \\
\text { - Cognitive interviews with } \\
\text { young adults } \\
\text { - Concept mapping with } \\
\text { providers/staff }\end{array}$ & $\begin{array}{l}\text { Evidence of the usability } \\
\text { and usefulness of the CNQ- } \\
\text { YP }\end{array}$ \\
\hline $\begin{array}{l}\text { Identify user and contextual } \\
\text { requirements } \\
\text { (the fabric) }\end{array}$ & $\begin{array}{l}\text { Ethnographic contextual } \\
\text { inquiry } \\
\text { - Guided tours with young } \\
\text { adults and providers/staff } \\
\text { from Lineberger } \\
\text { - Semi-structured interviews } \\
\text { with providers/staff from } \\
\text { external organizations }\end{array}$ & $\begin{array}{l}\text { User and contextual } \\
\text { requirements for NA-SB's } \\
\text { design and implementation }\end{array}$ \\
\hline $\begin{array}{l}\text { Design EBP and implementation } \\
\text { strategy prototypes based on user } \\
\text { and contextual requirements } \\
\text { (thread + fabric }+ \text { needle) }\end{array}$ & $\begin{array}{l}\text { Design team Workshops } \\
\text { - Workshop \#1 } \\
\text { - Workshop \#2 }\end{array}$ & $\begin{array}{l}\text { NA-SB prototypes and } \\
\text { anticipated implementation } \\
\text { strategies needed }\end{array}$ \\
\hline RESULT & & $\begin{array}{l}\text { NA-SB }+ \text { compilation of } \\
\text { relevant implementation } \\
\text { strategies }\end{array}$ \\
\hline
\end{tabular}

\section{Multidisciplinary design team}

In dissemination and implementation, stakeholder engagement is often limited or superficial[39-41]. In contrast, UCD demands an active and iterative approach to engagement, often with the same group of users reviewing prototypes at multiple time points[42]. Thus, at the beginning of the project, we convened an NA-SB design team comprised of key stakeholder groups. Throughout the project, the investigator team presented prototypes and other information to the design team and, based on their interactions with 
prototypes and collaborative discussion, made iterative improvements to NA-SB design and implementation strategies.

Design team members included researchers in cancer care delivery, patient-reported outcomes, UCD, and implementation science $(n=4)$ and prospective NA-SB users, including Lineberger clinical partners (oncologist; social worker/director of Lineberger's young adult program [ $n=2]$ ), and young adult representatives $(n=5)$ nominated by clinical partners. Nominees were primarily individuals who had previously expressed interest in research or advocacy activities related to young adult cancer and thus, would be more likely to consider the extensive and ongoing participation that joining the design team would entail.

To recruit young adult representatives for the design team, clinical partners connected young adults via email to the project lead (EH). EH provided them with materials including a project summary, a breakdown of their expected role and time commitment, and a brief summary of UCD. EH then met with each young adult interested in participating to discuss the project and develop rapport, then met with them all together to build group rapport. Young adult representatives received a one-time $\$ 150$ incentive for participation.

Young adult representatives included a racially and ethnically diverse group of 5 men and women. They represented different timepoints in their cancer trajectory, with one in active treatment, one in maintenance treatment, and the others in the survivorship phase. In addition to their lived experience with cancer, young adult representatives brought other areas of content expertise to the project, including instructional design and mental health service delivery.

(1) Review and refine prototypes (optimize the thread)

\section{Overview}

We conducted three rounds of usability testing to examine user interactions with the CNQ-YP: (1) an online survey assessing young adults' needs and preferences for a needs assessment using the CNQ-YP as a prototype for them to react to; (2) cognitive interviews[43] with young adults to triangulate survey data with in-depth evidence of their perceptions of the CNQ-YP's usability and usefulness; and (3) concept mapping[44] exercises focused on usefulness, in which young adult program providers mapped CNQ-YPidentified needs onto services/resources to address the needs.

\section{Young adult survey}

Objectives. To identify missing content, streamline redundant or low-priority content, and other usability and usefulness concerns.

Instrument. The survey instrument (Additional File 1) included three sections: (1) study information, consent, and demographic items (i.e., age, gender, clinical characteristics, social support, educational/vocational status, health insurance status), (2) the CNQ-YP in its original form, and (3) items assessing respondents' perception of the CNQ-YP. To assess general attitudes towards the tool, we used 
items from three Likert-type measures of feasibility, acceptability, and appropriateness[45]. We assessed usefulness through two Likert-type items asking (1) the extent to which respondents thought the CNQ-YP accurately captured their needs, and (2) the likelihood that they would use services or resources offered to them based on indicated needs. For each of these measures, we qualitatively probed respondents on usability and usefulness issues driving their concerns with the tool's feasibility, acceptability, or appropriateness.

Sample and Recruitment. To be included in the survey, we required participants $(n=100)$ to be age $18-30$ and have been diagnosed with cancer prior to survey administration. To ensure young adult participant diversity (race, ethnicity, age, geographic region, setting of care, etc.), we recruited through key contacts (i.e., leaders of young adult programs and advocacy groups in the United States identified by our clinical partners), social media (i.e., a series of Twitter messages shared by tagging relevant groups and hashtags), and our design team.

Procedure. We administered the survey through a secure online platform, Qualtrics (Provo, UT). On average, the survey took 15 minutes to complete.

Analysis. We used descriptive statistics for respondents' demographics, needs reported on the CNQ-YP tool, and perceptions of the CNQ-YP. To identify emergent themes regarding the CNQ-YP's usability and usefulness in free-text responses, we used template analysis[46].

\section{Cognitive interviews}

Objective. To triangulate survey data on CNQ-YP usability and usefulness through nuanced understanding of content, wording, or comprehension concerns.

Interview guide. With input from the design team, we developed the cognitive interview guide to encourage participants to "think aloud" as they read and reflected on the CNQ-YP itemset and probe them to comment on topics such as item content and wording, response options, format, length, comprehensiveness, repetitiveness, etc. (Additional File 2).

Sample and recruitment. To promote generalizability of cognitive interviews, we purposively sampled from among survey participants individuals who varied across select demographic characteristics (i.e., age, race, gender). We recruited young adults $(n=5)$ until we reached thematic saturation, i.e., when subsequent interviews did not generate new information regarding CNQ-YP's usability or usefulness.

Procedure. EH conducted one-hour cognitive interviews $(n=5)$ via Zoom, a video-conferencing platform. Interviews were audio-recorded. EH navigated the CNQ-YP through the Zoom screen-share function, soliciting participants' input on each item. At the end of each interview, EH summarized her takeaways with interviewees for the purposes of member checking[47].

Analysis. We inductively identified themes, noting concerns related to the CNQ-YP's usability and usefulness. We then created a table organizing participants' concerns within each of the identified 
themes for presentation to the design team during our first workshop (described later).

\section{Concept mapping}

Objective. To group needs assessed by the CNQ-YP by services/resources expected to address those needs.

Instrument. The design team approved changes to CNQ-YP content based on survey and cognitive interview results. We pre-loaded the resulting list of young adult needs into an online secure platform called Concept Systems Global Max @ (CSGM). CSGM included two concept mapping exercises: (1) sorting an electronic deck of cards, each containing a young adult need, into like categories (i.e., "followup domains") that could be addressed by the same service/resource, or other follow-up action (e.g., needs related to depression and anxiety might be grouped together as potentially addressable by referral to a mental health professional); and (2) rating needs on Likert-type response scales in terms of two key pragmatic properties: importance (i.e., severity of consequences if that need goes unmet) and actionability (i.e., likelihood that need can be met through a service or resource)[48].

Sample and recruitment. Concept mapping participants included cancer program providers (e.g., oncologists, nurses, and social workers) and staff (e.g., program managers and administrators) - i.e., the prospective NA-SB user group expected to have the most knowledge about service and resource delivery for this population. Recruitment through the key contacts established during survey recruitment was intended to achieve the minimum sample size of $n=15$ needed for concept mapping analyses[49].

Procedure. Participants accessed the web-based concept mapping exercises through emailed links to the project in CSGM. The exercises took approximately 30 minutes to complete.

Analysis. CSGM used hierarchical cluster analysis to characterize how participants grouped needs, creating several potential cluster maps based on proximity among needs, where proximal needs were more frequently grouped together as triggering the same follow-up action than distal ones, and 'go-zone graphs', in which needs are displayed as points on a quadrant in terms of their relative importance and actionability. Young adult design team representatives vetted results.

(2) Identify user and contextual requirements (understand and prepare the fabric)

\section{Overview}

To gather detailed information about prospective users and the context for NA-SB implementation, we used ethnography (i.e., guided tours and semi-structured interviews), a promising yet underused method for implementation research[50]. By documenting naturally-occurring user tasks and interactions among patients and providers through in-depth observation, ethnography provides rich data on implementation context[51, 52]. Ethnographic methods are relevant to UCD because they offer more nuanced understanding of users and context than traditional questionnaires or interviews, including novel insights on user tasks, attitudes, and interactions with their environment $[17,21,53]$. We used a novel UCD user and contextual factor framework[21] to guide data collection and analysis, combining ethnography and 
contextual inquiry. Data from this phase informed context modifications and identification of minimally necessary implementation strategies. Additional File 4 includes the Standards for Reporting Qualitative Research (SRQR) checklist adhered to for these data collection activities.

\section{Guided tours}

Objective. To capture contextual elements beyond just those which users can verbalize, including details and motivations that have become habitual or implicit to the tasks they perform[54].

Instrument. To promote the flexibility required for guided tours $[55,56]$, we identified potential questions based on four domains of Maguire et al.'s typology of user and contextual factors to consider in UCD from which we could choose: (1) user characteristics, (2) user tasks, (3) physical and technical environment, and (4) organizational environment[21] (Additional File 3).

Sample and recruitment. To capture the perspective of potential NA-SB implementers, we conducted guided tours with our clinical partners at Lineberger $(n=2)$. To capture the patient perspective, we conducted guided tours with young adults ages 18-30 receiving inpatient or outpatient care at Lineberger $(n=10)$. Our clinical partners at Lineberger facilitated the recruitment of young adults for guided tours by distributing a recruitment flyer and connecting EH via email to those interested.

Procedure. EH conducted four-hour guided tours with clinical partners as they completed clinical, administrative and other duties, asking questions about their tasks and thoughts. EH followed young adults and accompanying family members from the moment they entered the hospital for their outpatient appointments until the moment they exited, asking them questions as they interacted with their environment and healthcare professionals, while attempting to minimize participant disruptions. For inpatient guided tours, EH spent two hours with young adults receiving inpatient care. $\mathrm{EH}$ took extensive field notes and audio-recorded portions of the guided tours for which only consenting parties were present. We offered young adult participants a $\$ 50$ participation incentive.

Analysis. We used template analysis, identifying a priori themes based on Maguire's constructs and allowing for identification of additional themes[46]. Two study authors independently coded excerpts from guided tour field notes and interview transcriptions per Maguire constructs. For each domain, they collaboratively synthesized user and contextual factors and created a "translation table"[57], which translated factors into their implications for NA-SB design and implementation. For example, providers reported the importance of integrating new tools into the electronic medical record; we translated this into the requirement that NA-SB interface with Lineberger's electronic medical record. All requirements were vetted and prioritized by the design team during the second workshop (see description below).

\section{Semi-structured interviews}

Objective. To review findings from guided tours with external users and identify any areas of divergence or additional needs or contextual features, thus promoting generalizability of findings. 
Interview guide. With input from the design team, we developed a semi-structured interview guide based on Maguire's typology[21] and guided tour findings.

Sample. We conducted semi-structured interviews with the leaders of young adult programs and advocacy groups who had previously facilitated survey and concept mapping recruitment: program managers $(n=2)$, nurse navigators $(n=2)$, and patient navigators $(n=2)$ serving primarily young adults.

Procedure. EH conducted one-hour semi-structured telephone interviews. At the end of each interview, EH summarized major takeaways for member checking[47]. We audio-recorded and transcribed interviews verbatim.

Analysis. We analyzed interview data using template analysis[46].

(3) Design prototypes based on user and contextual requirements (thread the needle)

\section{Overview}

Through two three-hour workshops, the design team collaboratively redesigned the CNQ-YP (i.e., the EBP) with usability and usefulness in mind and redesigned Lineberger care processes (i.e., the context) to facilitate the tool's implementation and usefulness in routine care. This prototyping process - which relied on visual cues to digest user data with multiple user groups - represents a novel method for coproduction in implementation science. It resulted in NA-SB and a compilation of implementation strategies, each informed by context and designed to account for the other's characteristics.

\section{Design Team Workshop \#1}

After the survey, cognitive interviews, and concept mapping, we convened the NA-SB design team for a workshop in which we selected the most interpretable concept mapping cluster map (i.e., the cluster map with the highest face validity) and considered eliminating from the CNQ-YP needs that were relatively unimportant and not actionable. For example, the design team eliminated items assessing whether patients perceived their treatment staff as "approachable" or "friendly" based on concept mapping participants' low ratings of these items' actionability in terms of service or resource provision. The refinement of CNQ-YP items was further informed by usability and usefulness data from the survey and cognitive interviews. For example, the original CNQ-YP features similar items with different lookback periods (e.g., "since my diagnosis", "in the last month"); survey and cognitive interview participants felt these multiple reference periods were confusing and redundant given the tool's goal of addressing current needs through service provision. Thus, the design team anchored all items to needs present at the time of CNQ-YP completion and eliminated redundant content. In other cases, items were added based on user feedback on missing content (e.g., sexual health). Usability data also informed changes to the tool's display, instructions, and sequencing.

After grouping high-priority needs by follow-up domains, the design team identified services/resources at Lineberger which corresponded to each follow-up domain. We also anticipated implementation strategies needed to facilitate this kind of multidisciplinary service provision. The meeting resulted in a redesigned 
patient-reported outcome measure for use in NA-SB in which priority needs were grouped based on services/resources available at Lineberger to address those needs.

\section{Design Team Workshop \#2}

After soliciting user and contextual data through guided tours and interviews, we convened the design team for workshop \#2 during which we presented the ethnography translation table (developed from the guided tours and semi-structured interviews) alongside the NA-SB measure produced during design team workshop \#1. This allowed the design team to anticipate context modifications and necessary implementation strategies with respect to the redesigned tool. For example, the content of the tool might necessitate its administration by certain providers (e.g., social workers); staffing and workflow patterns may need modification to facilitate administration by social workers. Through popular UCD methods, 'personas' (i.e., generic representations of key user groups to convey users' needs to the design team,) and 'scenarios of use' (i.e., specific examples of how users, context, and NA-SB might interact)[21], we collaboratively determined the most salient requirements for NA-SB delivery, resolved discrepant requirements, and specified who will deliver the needs assessment, when, how often, and the materials and procedure that will be used to do so. Additionally, we used this workshop to co-design the bridge between identified needs and care delivery-i.e., making explicit the follow-up actions needed for each patient-reported need. Finally, we walked through Maguire's four domains to discuss potential remaining barriers to NA-SB-context fit and identify appropriate implementation strategies to address them. For example, some important user and contextual requirements could not be addressed through specification of NA-SB delivery (e.g., leadership buy-in); these were areas in which the application of strategies was deemed important for future implementation.

\section{Results}

To allow for more detailed focus on our methods, we have limited our reporting of results to key takeaways on how UCD may enable EBP-context-implementation strategy harmonization. More detailed results will be presented in future publications. Briefly, though, the methods described above culminated in an NA-SB prototype, including a redesigned patient-reported outcome measure and a protocol for its implementation. By ensuring NA-SB directly responded to features of users and context, we designed NASB for implementation, potentially minimizing the strategies needed to address misalignment that may have otherwise existed. Furthermore, we designed NA-SB for scale-up; by engaging users from other cancer programs across the country to identify points of contextual variation which would require flexibility in delivery, we created a tool not overly tailored to one unique context.

\section{Discussion}

To date, efforts to improve healthcare quality have focused on implementing EBPs as originally designed[58]. A potential consequence of this emphasis on EBPs has been resistance to considering the implications of their design for implementation into practice and an overemphasis on rigid conceptualizations of fidelity. Faced with EBPs that have been carefully tailored to unique contexts, implementation scientists often must facilitate their implementation with features ill-suited to the diverse 
scale-up contexts. In response, implementation scientists turn to implementation strategies that may further burden users. In fact, the NIH defines implementation science as "the scientific study of the use of strategies to adopt and integrate evidence-based health interventions into clinical and community settings"[59], a definition that we think overly narrows the scope of practice for implementation. In this paper, we assert that the pitfalls associated with cumbersome EBPs and implementation strategies may be avoided by attending equally to EBPs, contexts, and implementation strategies, through the application of UCD.

Just as embroidering requires compatible thread, fabric, and needle, implementation may be optimized by harmonizing EBP, context, and implementation strategies. We acknowledge that this analogy is imperfect; for example, some might regard embroidery as decoration or embellishment; on the contrary, our intention with this analogy is to convey the integration of the thread such that it becomes a part of the fabric itself. Despite its imperfection, the analogy is useful as it urges implementation scientists to attend equally to features of EBPs, context, and implementation strategies. Doing so has the potential to limit the challenges associated with complex EBPs and implementation strategies that burden stakeholders.

In this case example, usability testing elicited user concerns about the CNQ-YP that may have limited its uptake in practice, allowing our design team to redesign the CNQ-YP to maximize usability and usefulness. For example, through concept mapping, providers identified needs assessed by the CNQ-YP which, as originally written, could not be addressed with available services/resources (e.g., "I feel frustrated"); assessing such unactionable needs would have produced additional burden for users, without improving care. Through the survey and cognitive interviews, young adults identified important missing content (e.g., sexual health), and other areas in which the CNQ-YP's content, length, wording, and response format were unacceptable. By addressing these usability and usefulness concerns upfront, we designed a tool to be more feasible, acceptable, and appropriate to users.

Considering EBP characteristics like usability and usefulness in a vacuum may compromise implementation and burden stakeholders. To avoid these concerns, we leveraged UCD contextual inquiry methods to describe both NA-SB's specific implementation context (i.e., Lineberger) as well as its broader future scale-up context (i.e., other young adult cancer programs in the United States). To explore context, UCD offers frameworks such (e.g., Maguire's framework), as well as questionnaires (e.g., System Usability Scale[60]), and a menu of methods (e.g., diary keeping, user surveys, etc.[21]) compatible with others used by implementation scientists in the assessment of implementation determinants. Despite some overlap in UCD and implementation science methods, UCD goes further than traditional barriers/facilitators assessment by embedding users more deeply in the process. In this case example, we used ethnographic contextual inquiry to obtain a detailed understanding of users and context. Additionally, we went further than traditional barriers/facilitators assessments by engaging users in analysis to promote shared understanding of context: our design team reviewed ethnography findings to ensure that the user interpretation of context remained central, as opposed to relying solely on the researcher's interpretation of contextual data. 
UCD also provides methods for translating user and contextual factors into user and contextual requirements-i.e., usability and usefulness determinants[21]. Translating contextual factors into contextual requirements using UCD requirements engineering approaches (e.g., translation tables, 'personas', and 'scenarios-of-use') could help implementation scientists prioritize implementation determinants by focusing attention on the critical subset of contextual factors that influence EBP usability and usefulness[13]. In this case example, the ethnography provided valuable source data for workshop materials, helping us to leverage design team expertise to identify these usability determinants and prioritize contextual features to target with EBP redesign, context preparation, or implementation strategies. During design team workshop \#2, we presented several alternative scenarios-of-use, or simple descriptions of plausible user interactions with NA-SB, to inform the specification of NA-SB delivery. These scenarios provide user- and task-oriented information about the context in which an EBP has to operate[61], and also offer concrete examples for design team members to react to. For example, scenarios helped our design team walk through different patient visit types (e.g., just infusion versus infusion + clinical visit) to ensure that design decisions about staffing and timing for needs assessment administration suited the range of potential appointments.

We used UCD to enhance the usability and usefulness of NA-SB and reduce the number of implementation strategies needed to embed the tool in routine care. However, where EBP and context diverge, UCD can help tailor strategies which make EBP and context more compatible. In this case example, we anticipated areas where NA-SB provision may clash with user or contextual requirements, some of which could not be addressed by EBP redesign or context preparation. For example, NA-SB - a tool that spans across multiple domains of care - will require the cooperation of multiple departments and disciplines; although users are more likely to buy into a usable and useful tool $[62,63]$ and engaging users in its development likely generated some buy-in, additional implementation strategies targeting cross-department buy-in may be required. These remaining gaps in EBP-context fit inform the selection of strategies to promote NA-SB implementation. Leveraging UCD to identify user and contextual requirements and tailor implementation strategies addresses an articulated need in the field[14,64] and complements approaches for selecting and tailoring strategies that have recently been proposed in the implementation science literature[13]. Future work will assess the extent to which UCD minimizes the need for complex implementation strategies or, when needed, aids in the tailoring of strategies that are contextually appropriate and minimally burdensome.

As demonstrated by this case example, UCD can help implementation scientists to operationalize the field's commitment to stakeholder engagement. For example, establishing a design team upfront ensured that users remained central throughout NA-SB development and implementation planning. Design team members offered key insights to inform data collection (e.g., review of instruments), data analysis (e.g., selection of concept mapping cluster map; prioritization of user and contextual requirements), and, ultimately, NA-SB and implementation strategy design. Further, design team members proved critical to the recruitment of users for usability testing and ethnographic data collection. UCD also offers methods for translating user feedback into design decisions. For example, the use 'scenarios-of-use', allowed our design team to translate ethnographic data into NA-SB design features, in a way that group discussion 
without such engagement methods may not have. Finally, UCD demands an iterative approach to user engagement, often with the same group of users reviewing prototypes at multiple time points; this type of iteration may be a key moderator in the relationship between stakeholder engagement and improved EBP design[42]. The extent which this holds true for implementation is a topic that warrants further exploration.

Applying UCD to implementation science has notable challenges. Embedding the extensive engagement UCD requires can sometimes be costly and time intensive. Additionally, this level of engagement places issues of sampling and recruitment at the forefront. For example, the UCD process hinges on complex decisions about who counts as a user and which individuals accurately represent users more broadly. Prioritizing divergent feedback from multiple user groups[65], or weighing the relative importance of user feedback with the feasibility of design solutions, may not always be straightforward. Inexpert application of UCD methods may lead to 'feature creep', in which new ideas are incorporated into the EBP without careful consideration and evaluation of the effects of the added features. UCD's emphasis on iterative design thinking and local insights may also raise concerns about diminishing fidelity as EBPs are recurrently revised to better align with context outside of the controlled environment where the EBP was originally designed and tested. Finally, implementation scientists may struggle to shoulder the challenges associated with incorporating new disciplines into already multidisciplinary teams and projects (e.g., reconciling terminology and frameworks). In addition to such practical differences, there may be fundamental philosophical differences between the two fields. In general, implementation science focuses more-so on the EBP and UCD on the users; where there is divergence between what is best for the EBP and what is best for the user, reconciling these competing viewpoints may be difficult. However, if implementation scientists are to leverage key insights from other disciplines, they must continue to surmount such roadblocks to knowledge integration.

\section{Conclusions}

Implementing change in dynamic healthcare settings is complex; understanding the nuances of implementation requires a multimodal, multidisciplinary purview. To this end, implementation scientists have borrowed knowledge and approaches from systems science[66, 67], organizational studies[68], cultural adaptation[69], community-based participatory research[70], behavioral psychology[71], and quality improvement[72], just to name a few. We argue that UCD methods like usability testing, ethnographic contextual inquiry, and design team prototyping can join the list of approaches available to implementation scientists. This may first require investigation of where UCD and implementation science converge and diverge. Fortunately, efforts to this effect are currently underway[73]. While points of divergence may represent barriers to integration of the two fields, they may also represent important new insights and approaches for implementation scientists to consider.

Just as embroidery requires the alignment of thread, fabric, and needle, EBP implementation and sustainment requires harmonizing EBP, context, and implementation strategies. The importance of each of these has been acknowledged; however, methods for understanding the dynamic interplay among 
them and optimizing each with respect to the other two are lacking. UCD offers methods and approaches for achieving this. Future research should explore the utility of collaborating with UCD experts or embedding UCD approaches in implementation research[73]. In particular, we argue that UCD's potential for promoting harmonization among EBP, context, and implementation should be tested empirically, work that is currently underway[74]. To the extent that UCD helps facilitate this harmonization, it will advance us towards the field's goal of bridging the gap between research and practice.

\section{Abbreviations}

EBP

Evidence-based practice

UCD

User-Centered Design

NA-SB

Needs Assessment \& Service Bridge

CNQ-YP

Cancer Needs Questionnaire- Young People

CSGM

Concept Systems GlobalMax@

\section{Declarations}

\section{Ethics approval and consent to participate}

This study was approved by the University of North Carolina's Institutional Review Board (19-0255).

\section{Consent for publication}

Not applicable

\section{Availability of data and materials}

Data collection instruments are available as additional files.

\section{Competing interests}

The authors declare that they have no competing interests.

\section{Funding}

Ms. Haines' effort was supported by funding from UNC Lineberger's University Cancer Research Fund. Dr. Dopp is an investigator with the Implementation Research Institute, at the George Warren Brown School of Social Work, Washington University in St. Louis; through an award from the National Institutes of 
Mental Health (5R25MH08091607) and the Department of Veterans Affairs, Health Services Research \& Development Service, Quality Enhancement Research Initiative (QUERI). Dr. Birken's effort was supported by the National Center for Advancing Translational Sciences, National Institutes of Health, through Grant KL2TR002490. The content is solely the responsibility of the authors and does not necessarily represent the official views of the $\mathrm{NIH}$.

\section{Authors' contributions}

All authors (ERH, AD, AL, HW, MB, GV, DH, SB) were involved in manuscript conceptualization, drafting, and editing. All authors read and approved the final manuscript.

Acknowledgements

Not applicable

\section{References}

1. Chambers $D A$, Glasgow RE, Stange KC: The dynamic sustainability framework: addressing the paradox of sustainment amid ongoing change. Implementation Science 2013, 8(1):117.

2. Pfadenhauer LM, Mozygemba K, Gerhardus A, Hofmann B, Booth A, Lysdahl KB, Tummers M, Burns $J$, Rehfuess EA: Context and implementation: A concept analysis towards conceptual maturity. Zeitschrift fur Evidenz, Fortbildung und Qualitat im Gesundheitswesen 2015, 109(2):103-114.

3. Rabin BA, Brownson RC: Terminology for dissemination and implementation research. Dissemination and implementation research in health: Translating science to practice 2017, 2:19-45.

4. Chaffin M, Silovsky JF, Funderburk B, Valle LA, Brestan EV, Balachova T, Jackson S, Lensgraf J, Bonner BL: Parent-child interaction therapy with physically abusive parents: efficacy for reducing future abuse reports. Journal of consulting and clinical psychology 2004, 72(3):500.

5. Lyon AR, Koerner K: User-Centered Design for Psychosocial Intervention Development and Implementation. Clinical Psychology: Science and Practice 2016, 23(2):180-200.

6. Chambers DA, Norton WE: The adaptome: advancing the science of intervention adaptation. American journal of preventive medicine 2016, 51(4):S124-S131.

7. Greenhalgh T, Robert G, Macfarlane F, Bate P, Kyriakidou O: Diffusion of innovations in service organizations: systematic review and recommendations. The Milbank Quarterly 2004, 82(4):581-629.

8. Titler MG: The evidence for evidence-based practice implementation. In: Patient safety and quality: An evidence-based handbook for nurses. edn.: Agency for Healthcare Research and Quality (US); 2008.

9. Nilsen P, Bernhardsson S: Context matters in implementation science: a scoping review of determinant frameworks that describe contextual determinants for implementation outcomes. $B M C$ health services research 2019, 19(1):189. 
10. Proctor EK, Powell BJ, McMillen JC: Implementation strategies: recommendations for specifying and reporting. Implementation Science 2013, 8(1):139.

11. Powell BJ, Waltz TJ, Chinman MJ, Damschroder LJ, Smith JL, Matthieu MM, Proctor EK, Kirchner JE: A refined compilation of implementation strategies: results from the Expert Recommendations for Implementing Change (ERIC) project. Implementation Science 2015, 10(1):21.

12. Brownson RC, Colditz GA, Proctor EK: Chapter 15 Implementation Strategies in Dissemination and implementation research in health: translating science to practice: Oxford University Press; 2018.

13. Powell BJ, Beidas RS, Lewis CC, Aarons GA, McMillen JC, Proctor EK, Mandell DS: Methods to Improve the Selection and Tailoring of Implementation Strategies. The Journal of Behavioral Health Services \& Research 2017, 44(2):177-194.

14. Grol R, Wensing M, Eccles M, Davis D: Improving patient care: the implementation of change in health care: John Wiley \& Sons; 2013.

15. Kislov R, Pope C, Martin GP, Wilson PM: Harnessing the power of theorising in implementation science. Implementation Science 2019, 14(1):103.

16. Eccles MP, Armstrong D, Baker R, Cleary K, Davies H, Davies S, Glasziou P, llott I, Kinmonth A-L, Leng $\mathrm{G}$ et al: An implementation research agenda. Implementation Science 2009, 4(1):18.

17. Johnson $\mathrm{CM}$, Johnson TR, Zhang J: A user-centered framework for redesigning health care interfaces. Journal of Biomedical Informatics 2005, 38(1):75-87.

18. Standardization IOf: ISO 9241-11: Ergonomic Requirements for Office Work with Visual Display Terminals (VDTs): Part 11: Guidance on Usability; 1998.

19. Nielsen J: Usability engineering: Elsevier; 1994.

20. Lyon AR, Bruns EJ: User-Centered Redesign of Evidence-Based Psychosocial Interventions to Enhance Implementation-Hospitable Soil or Better Seeds? JAMA psychiatry 2018.

21. Maguire M: Methods to support human-centred design. International Journal of Human-Computer Studies 2001, 55(4):587-634.

22. Witteman HO, Dansokho SC, Colquhoun H, Coulter A, Dugas M, Fagerlin A, Giguere AMC, Glouberman S, Haslett L, Hoffman A et al: User-centered design and the development of patient decision aids: protocol for a systematic review. Systematic Reviews 2015, 4(1):11.

23. Dopp AR, Parisi KE, Munson SA, Lyon AR: A glossary of user-centered design strategies for implementation experts. Translational behavioral medicine 2018.

24. Proctor E, Silmere H, Raghavan R, Hovmand P, Aarons G, Bunger A, Griffey R, Hensley M: Outcomes for implementation research: conceptual distinctions, measurement challenges, and research agenda. Administration and Policy in Mental Health and Mental Health Services Research 2011, 38(2):65-76.

25. U.S. Department of Health and Human Services CfDCaPaNCI: U.S. Cancer Statistics Working Group. U.S. Cancer Statistics Data Visualizations Tool, based on November 2018 submission data (19992016). In.; June 2019. 
26. Keegan TH, Lichtensztajn DY, Kato I, Kent EE, Wu X-C, West MM, Hamilton AS, Zebrack B, Bellizzi KM, Smith AW: Unmet adolescent and young adult cancer survivors information and service needs: a population-based cancer registry study. Journal of Cancer Survivorship 2012, 6(3):239-250.

27. Dyson GJ, Thompson K, Palmer S, Thomas DM, Schofield P: The relationship between unmet needs and distress amongst young people with cancer. Supportive Care in Cancer 2012, 20(1):75-85.

28. Sawyer SM, McNeil R, McCarthy M, Orme L, Thompson K, Drew S, Dunt D: Unmet need for healthcare services in adolescents and young adults with cancer and their parent carers. Supportive Care in Cancer 2017, 25(7):2229-2239.

29. Zebrack BJ, Corbett V, Embry L, Aguilar C, Meeske KA, Hayes-Lattin B, Block R, Zeman DT, Cole S: Psychological distress and unsatisfied need for psychosocial support in adolescent and young adult cancer patients during the first year following diagnosis. Psychooncology 2014, 23(11):1267-1275.

30. Zebrack BJ, Block R, Hayes-Lattin B, Embry L, Aguilar C, Meeske KA, Li Y, Butler M, Cole S:

Psychosocial service use and unmet need among recently diagnosed adolescent and young adult cancer patients. Cancer 2013, 119(1):201-214.

31. Smith A, Parsons H, Kent E, Bellizzi K, Zebrack B, Keel G, Lynch C, Rubenstein M, Keegan T: Unmet Support Service Needs and Health-Related Quality of Life among Adolescents and Young Adults with Cancer: The AYA HOPE Study. Frontiers in Oncology 2013, 3(75).

32. Smits-Seemann RR, Kaul S, Zamora ER, Wu YP, Kirchhoff AC: Barriers to follow-up care among survivors of adolescent and young adult cancer. Journal of Cancer Survivorship 2017, 11(1):126-132.

33. Gardner MH, Barnes MJ, Bopanna S, Davis CS, Cotton PB, Heron BL, Henninger A, Alva E, Gleason MW, Whelan KF et al: Barriers to the Use of Psychosocial Support Services Among Adolescent and Young Adult Survivors of Pediatric Cancer. Journal of Adolescent and Young Adult Oncology 2014, 3(3):112-116.

34. Barakat LP, Galtieri LR, Szalda D, Schwartz LA: Assessing the psychosocial needs and program preferences of adolescents and young adults with cancer. Supportive Care in Cancer 2016, 24(2):823-832.

35. Clinton-McHarg T, Carey M, Sanson-Fisher R, D'Este C, Shakeshaft A: Preliminary development and psychometric evaluation of an unmet needs measure for adolescents and young adults with cancer: the Cancer Needs Questionnaire - Young People (CNQ-YP). Health and Quality of Life Outcomes 2012, 10(1):13.

36. Clinton-McHarg T, Carey M, Sanson-Fisher R, Shakeshaft A, Rainbird K: Measuring the psychosocial health of adolescent and young adult (AYA) cancer survivors: a critical review. Health and Quality of Life Outcomes 2010, 8(1):25.

37. Howell D, Molloy S, Wilkinson K, Green E, Orchard K, Wang K, Liberty J: Patient-reported outcomes in routine cancer clinical practice: a scoping review of use, impact on health outcomes, and implementation factors. Annals of Oncology 2015, 26(9):1846-1858.

38. Papadakos JK, Charow RC, Papadakos CJ, Moody LJ, Giuliani ME: Evaluating cancer patientreported outcome measures: Readability and implications for clinical use. Cancer 2019, 125(8):1350- 
1356.

39. Oetzel JG, Zhou C, Duran B, Pearson C, Magarati M, Lucero J, Wallerstein N, Villegas M: Establishing the psychometric properties of constructs in a community-based participatory research conceptual model. American Journal of Health Promotion 2015, 29(5):e188-e202.

40. Brownson RC, Jacobs JA, Tabak RG, Hoehner CM, Stamatakis KA: Designing for dissemination among public health researchers: findings from a national survey in the United States. American journal of public health 2013, 103(9):1693-1699.

41. Holt CL, Chambers DA: Opportunities and challenges in conducting community-engaged dissemination/implementation research. Translational behavioral medicine 2017, 7(3):389-392.

42. Lai J, Honda T, Yang MC: A study of the role of user-centered design methods in design team projects. Artificial Intelligence for Engineering Design, Analysis and Manufacturing 2010, 24(3):303316.

43. DeMaio TJ, Rothgeb JM: Cognitive interviewing techniques: In the lab and in the field. In: Answering questions: Methodology for determining cognitive and communicative processes in survey research. edn. San Francisco, CA, US: Jossey-Bass; 1996: 177-195.

44. Trochim W: The reliability of concept mapping. In: annual conference of the American Evaluation Association: 1993; 1993.

45. Weiner BJ, Lewis CC, Stanick C, Powell BJ, Dorsey CN, Clary AS, Boynton MH, Halko H: Psychometric assessment of three newly developed implementation outcome measures. Implementation Science 2017, 12(1):108.

46. King N: Using templates in the thematic analysis of text. Essential guide to qualitative methods in organizational research 2004, 2:256-270.

47. Carlson JA: Avoiding traps in member checking. The qualitative report 2010, 15(5):1102-1113.

48. Lewis CC, Weiner BJ, Stanick C, Fischer SM: Advancing implementation science through measure development and evaluation: a study protocol. Implementation Science 2015, 10(1):102.

49. Kane M, Trochim WM: Concept mapping for planning and evaluation: Sage Publications, Inc; 2007.

50. Weiner BJ, Amick HR, Lund JL, Lee S-YD, Hoff TJ: Review: Use of Qualitative Methods in Published Health Services and Management Research: A 10-Year Review. Medical Care Research and Review 2010, 68(1):3-33.

51. Carnevale FA, Macdonald ME, Bluebond-Langner M, McKeever P: Using participant observation in pediatric health care settings: ethical challenges and solutions. Journal of Child Health Care 2008, 12(1):18-32.

52. Hammersley M: Reading ethnographic research: a critical guide [Internet]. In.: London, UK: Longman; 1990.

53. Locatelli SM, Turcios S, LaVela SL: Optimizing the Patient-Centered Environment: Results of Guided Tours With Health Care Providers and Employees. HERD: Health Environments Research \& Design Journal 2015, 8(2):18-30. 
54. Daae J, Boks C: A classification of user research methods for design for sustainable behaviour. Journal of Cleaner Production 2015, 106:680-689.

55. Balbale SN, Locatelli SM, LaVela SL: Through Their Eyes: Lessons Learned Using Participatory Methods in Health Care Quality Improvement Projects. Qualitative Health Research 2015, 26(10):1382-1392.

56. Brown L, Durrheim K: Different kinds of knowing: Generating qualitative data through mobile interviewing. Qualitative Inquiry 2009, 15(5):911-930.

57. Van Velsen L, Wentzel J, Van Gemert-Pijnen JEWC: Designing eHealth that Matters via a Multidisciplinary Requirements Development Approach. JMIR Research Protocols 2013, 2(1):e21.

58. Dixon-Woods M: How to improve healthcare improvement-an essay by Mary Dixon-Woods. $\mathrm{bmj}$ 2019, 367:15514.

59. Dissemination and implementation research in health (R01) NIH funding opportunity: PAR-19-274. NIH grant funding opportunities. In.: Department of Health and Human Services; 2018.

60. Brooke J: SUS-A quick and dirty usability scale. Usability evaluation in industry 1996, 189(194):4-7.

61. Battle L, Taylor DH: Sharing the Vision= Designs that Get Built.

62. Jensen-Doss A, Hawley KM: Understanding barriers to evidence-based assessment: Clinician attitudes toward standardized assessment tools. Journal of Clinical Child \& Adolescent Psychology 2010, 39(6):885-896.

63. Ivatury SJ, Hazard-Jenkins HW, Brooks GA, McCleary NJ, Wong SL, Schrag D: Translation of PatientReported Outcomes in Oncology Clinical Trials to Everyday Practice. Annals of Surgical Oncology 2019.

64. Wensing M, Bosch M, Grol R, Straus S, Tetroe J, Graham I: Knowledge translation in health care: moving from evidence to practice. 2009.

65. Oliver K, Kothari A, Mays N: The dark side of coproduction: do the costs outweigh the benefits for health research? Health Research Policy and Systems 2019, 17(1):33.

66. Zimmerman L, Lounsbury DW, Rosen CS, Kimerling R, Trafton JA, Lindley SE: Participatory system dynamics modeling: Increasing stakeholder engagement and precision to improve implementation planning in systems. Administration and Policy in Mental Health and Mental Health Services Research 2016, 43(6):834-849.

67. LUKE DA, MORSHED AB, MCKAY VR, COMBS TB: Systems science methods in dissemination and implementation research. In: Dissemination and implementation research in health: translating science to practice. edn.: Oxford University Press; 2018: 157-174.

68. Birken SA, Bunger AC, Powell BJ, Turner K, Clary AS, Klaman SL, Yu Y, Whitaker DJ, Self SR, Rostad WL: Organizational theory for dissemination and implementation research. Implementation Science 2017, 12(1):62.

69. Cabassa LJ, Baumann AA: A two-way street: bridging implementation science and cultural adaptations of mental health treatments. Implementation Science 2013, 8(1):90. 
70. Wallerstein N, Duran B: Community-Based Participatory Research Contributions to Intervention Research: The Intersection of Science and Practice to Improve Health Equity. American Journal of Public Health 2010, 100(S1):S40-S46.

71. Michie S, Johnston M: Theories and techniques of behaviour change: Developing a cumulative science of behaviour change. Health Psychology Review 2012, 6(1):1-6.

72. Balasubramanian BA, Cohen DJ, Davis MM, Gunn R, Dickinson LM, Miller WL, Crabtree BF, Stange $\mathrm{KC}$ : Learning Evaluation: blending quality improvement and implementation research methods to study healthcare innovations. Implementation Science 2015, 10(1):31.

73. Dopp AR, Parisi KE, Munson SA, Lyon AR: Integrating implementation and user-centred design strategies to enhance the impact of health services: protocol from a concept mapping study. Health research policy and systems 2019, 17(1):1.

74. Lyon AR, Munson SA, Renn BN, Atkins DA, Pullmann MD, Friedman E, Areán PA: Human-centered design to improve implementation of evidence-based psychotherapies in low-resource communities: UW ALACRITY Center Methods Core protocol. Journal of Medical Internet Research in press.

75. Damschroder LJ, Aron DC, Keith RE, Kirsh SR, Alexander JA, Lowery JC: Fostering implementation of health services research findings into practice: a consolidated framework for advancing implementation science. Implementation science 2009, 4(1):50.

76. Lyon AR, Coifman, J., Cook, H., Liu, F., Ludwig, K., Dorsey, S., Koerner, K., Munson, S., \& McCauley, E. : The Cognitive-though for Implementation Strategies (CWIS): A pragmatic methodology for assessing strategy usability. In: 11th Annual Conference on the Science of Dissemination and Implementation: 2018; Washington, DC, 2018.

\section{Additional Files}

\section{Additional File 1:}

This file contains the survey instrument used for the online survey of AYAs, including demographic questions, questions from the Cancer Needs Questionnaire-Young People tool, and questions surrounding the tool's usability and usefulness. (DOCX $50 \mathrm{~kb}$ )

\section{Additional File 2:}

This file contains the cognitive interview guide, including the original Cancer Needs Questionnaire-Young People. (DOCX $50 \mathrm{~kb}$ )

\section{Additional File 3:}

This file contains Maguire et al.'s framework of user and contextual factors to consider in User-Centered Design. The file also includes example questions within each domain of Maguire et al.'s framework used during ethnographic contextual inquiry (i.e., guided tours and semi-structured interviews). (DOCX $21 \mathrm{~kb}$ ) 
Additional File 4:

This file contains the Standards for Reporting Qualitative Research (SRQR) checklist. (DOCX $39 \mathrm{~kb}$ )

Figures

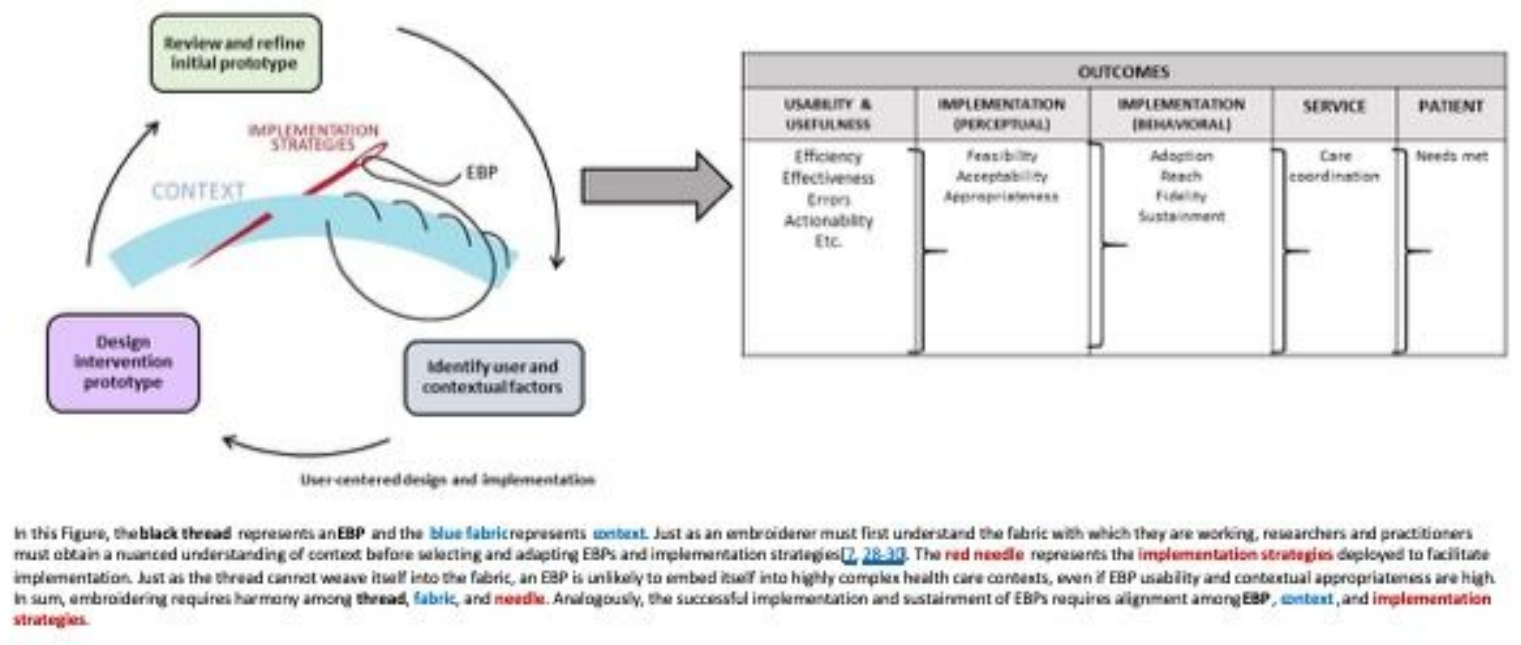

Figure 1

User-centered design and implementation 


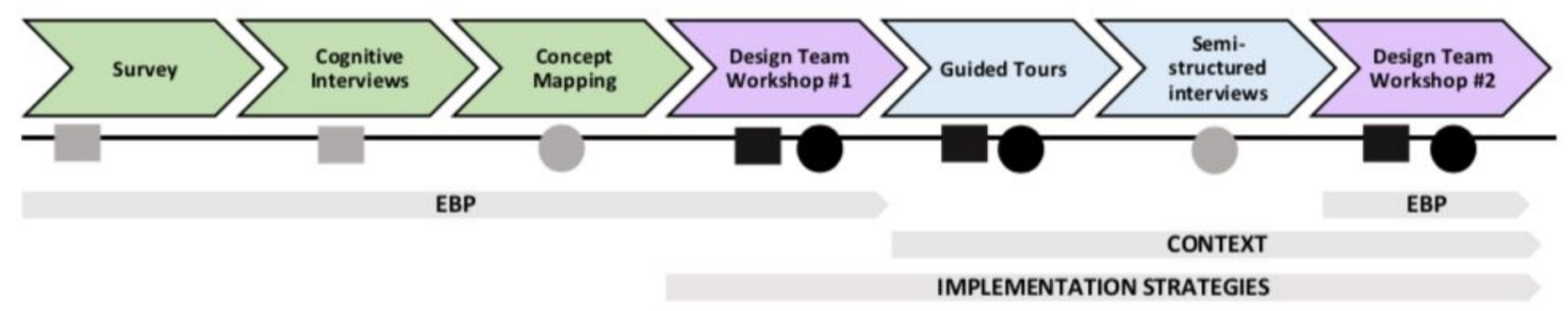

\section{Figure 2 legend}

External young adults Lineberger young adults

$\sum$ UCD STEP 1: Review and refine EBP prototype

$\sum$ UCD STEP 2: Identify user and contextual requirements

$\sum$ UCD STEP 3: Design EBP and implementation strategy prototypes
Figure 2 demonstrates the iterative engagement of both Lineberger and external users to harmonize, EBP, context, and implementation strategies. External users were engaged in the review and refinement of the CNQ-YP to avoid overtailoring EBP to one unique context (i.e., to promote generalizability). Because guided tours would inform preparation of the implementation context (i.e.. Lineberger), Lineberger users were engaged. Semi-structured interviews with external providers were then conducted to explore differences across contexts (i.e., to inform future scale-up in other cancer programs). The design team was comprised of Lineberger users to allow for in-person collaboration to produce design solutions highly applicable to Lineberger.

Figure 2

Data collection timeline and users engaged

\section{Supplementary Files}

This is a list of supplementary files associated with this preprint. Click to download.

- AdditionalFile1.docx

- AdditionalFile4.docx

- AdditionalFile3.docx

- AdditionalFile2.docx 